\title{
Easyreporting simplifies the implementation of Reproducible Research layers in R software
}

\author{
Dario Righelli $\oplus^{1,2 *}$, Claudia Angelini ${ }^{2 *}$ \\ 1 Department of Statistical Sciences, University of Padova, Padua, Italy, 2 Istituto per le Applicazioni del \\ Calcolo "Mauro Picone", National Research Council, Naples, Italy \\ *d.righelli@ na.iac.cnr.it (DR); c.angelini@iac.cnr.it (CA)
}

\section{Abstract}

During last years "irreproducibility" became a general problem in omics data analysis due to the use of sophisticated and poorly described computational procedures. For avoiding misleading results, it is necessary to inspect and reproduce the entire data analysis as a unified product. Reproducible Research (RR) provides general guidelines for public access to the analytic data and related analysis code combined with natural language documentation, allowing third-parties to reproduce the findings. We developed easyreporting, a novel R/Bioconductor package, to facilitate the implementation of an RR layer inside reports/tools. We describe the main functionalities and illustrate the organization of an analysis report using a typical case study concerning the analysis of RNA-seq data. Then, we show how to use easyreporting in other projects to trace $R$ functions automatically. This latter feature helps developers to implement procedures that automatically keep track of the analysis steps. Easyreporting can be useful in supporting the reproducibility of any data analysis project and shows great advantages for the implementation of R packages and GUls. It turns out to be very helpful in bioinformatics, where the complexity of the analyses makes it extremely difficult to trace all the steps and parameters used in the study.

Published: May 10, 2021

Peer Review History: PLOS recognizes the benefits of transparency in the peer review process; therefore, we enable the publication of all of the content of peer review and author responses alongside final, published articles. The editorial history of this article is available here: https://doi.org/10.1371/journal.pone.0244122

Copyright: @ 2021 Righelli, Angelini. This is an open access article distributed under the terms of the Creative Commons Attribution License, which permits unrestricted use, distribution, and reproduction in any medium, provided the original author and source are credited.

Data Availability Statement: All Supplementary Files and their source codes are available at https:// github.com/drighelli/easyreporting_supplementary.

Funding: The work has been partially supported by the Regione Campania Project ADViSE assigned to

\section{Introduction}

Due to accidental mistakes or misusage of sophisticated computational methods, many research findings in omics science are considered false (or partially incorrect) [1]. Moreover, in several cases, published results are not entirely reproducible due to the lack of information. For example, the analysis of the massive amount of omics data produced by high-throughput technologies requires combining several different methodologies from the preprocessing, data cleaning, and normalization to the downstream analysis. Therefore, it becomes challenging to trace all the steps and the parameters used within a complete analysis. Consequently, the lack of details, such as user-parameter or subtle data manipulation made with small code lines not reported in the material and methods sections of a manuscript, can lead to findings that are not reproducible. To prevent misleading results, several authors suggested adopting some best practices [2-4] that should help in publishing reproducible results. Nevertheless, the proposed approaches can be time-consuming and require significant effort by researchers. Therefore, to 
Dr. Claudia Angelini. The funders had no role in study design, data collection and analysis, decision to publish, or preparation of the manuscript. There was no additional external funding received for this study.

Competing interests: The authors have declared that no competing interests exist. fully exploit the advantages of Reproducible Research (RR), it is still necessary to provide tools that can trace all the details using automatic procedures $[5,6]$.

Recently, the scientific community proposed several approaches to support RR by developing tools that require a lower cost in terms of time and efforts to be used [5-9]. Among the different approaches, one common idea is to describe the steps with an analysis report built up as a mixture of natural language sentences along with computational language and graphical outputs. This document should include: i) the analyzed data, ii) the Code Chunks (CCs), iii) results and intermediate outputs (as tables and figures), and iv) all information that can enhance the work comprehensibility and reproducibility. Using human-readable reports instead of other procedures (for example, virtualization solutions such as docker containers) have the advantage that the final document can be easily understood by non-expert users, whereas docker containers require computationally experienced users. Moreover, a humanreadable report can be enriched with comments and favors knowledge transfer. Nevertheless, the two approaches are complementary and can be combined to achieve full reproducibility in terms of input/output of each algorithm/function and the possibility to re-create a computational environment that does not depend on specific user installations.

The $\mathrm{R}$ community proposed several solutions based on the literate statistical programming, like sweave [10], knitr and rmarkdown [11]. Within this framework, the authors can release a data analysis as a human-readable document that incorporates data, computational methods (including the short lines of code that are often omitted in a high-level description of the computational procedure), user-parameters, tables, and figures. Moreover, this report is automatically updated each time the analyst introduces some workflow changes to preserve complete reproducibility. R Studio (https://rstudio.com) already contains several functionalities that can help an analyst compiling detailed reports. Other R packages, such as Drake [12], go through the same directions.

Even though rmarkdown is very popular and easy to use inside the R community for writing step-by-step analysis reports, its usability when developing automated tools as Graphical User Interfaces (GUI) or packages is limited. Despite several efforts, incorporating a RR layer in other software and automatically tracing all the steps performed during a point-and-click analysis is still challenging. In the past, we proposed a solution with the RNASeqGUI [13, 14] project. RNASeqGUI is a GUI for analyzing RNA-seq data that automatically traces the analysis steps and reports them in a unique report. Although very useful, this solution did not allow the user to add personal comments, a particularly relevant requisite for knowledge transfer. Moreover, its implementation was time-consuming.

In light of these reasons, we developed easyreporting, a R/Bioconductor package allowing us to construct reports in different formats (i.e. HTML, PDF) that automatically incorporate comments with data, code, plots, and tables. In this work, we describe the easyreporting class and its methods. Then, we show i) how easyreporting can be used to generate user analysis reports and ii) how easyreporting can be used to implement packages and GUIs that automatically trace their functions and produce an analysis report. This latter feature makes easyreporting a particularly relevant and practical tool to improve user-friendly software. Moreover, the same approach can be used to create novel R packages or to trace user-defined functions.

\section{Materials and methods}

\section{Implementation}

Easyreporting is an open-source R/Bioconductor package aimed to 1) support analysts to speed up the compilation of their analysis reports and 2) help developers to integrate a RR 
layer inside their R software products (such as GUIs and packages). While the first aim can also be easily achieved using other similar tools, the latter constitutes one of the main advantages of our solution. In such a way, thanks to minimal efforts on the developers' side, the enduser can obtain an rmarkdown file that incorporates the source code generated during the analysis with the user-friendly tools. Once compiled, this document can then be published as supplementary material of a scientific article, helping the interested community to reproduce the computational part of the work entirely, as suggested in [15]. Moreover, the document can be easily organized into sections, describing different analysis steps, and enriched with natural language comments, making the report more explainable to increase the knowledge transfer.

\section{General description and initialization}

Easyreporting is structured as an S4 class representing a schematic view of a rmarkdown file (see Fig 1). Thanks to easyreporting, an analysis report can be seen as a particular instance of the package class, where the attributes represent the report characteristics. Within this class, the available methods are useful for attribute manipulations and for inserting comments and organizing section titles inside the report.

When easyreporting is used to create a report for novel analysis, the analyst needs to initialize an instance of the easyreporting class with the easyreporting() constructor function, passing as mandatory arguments the path and the name of the report file accompanied by its title. Optionally, it is possible to specify one or more authors' names (with emails, affiliations, affiliation websites, ORCIDs, and personal websites, like in a publication). Additionally, during the class creation, it is possible to define a bibliography latex file through the bibliography argument, that will be compiled as reference list at the end of the report (see Table 1).

In this way, each analysis/project is uniquely associated with a specific easyreporting instance, and hence to the corresponding rmarkdown file. The initialization step is transparent to any software user since the developers handle the tool's back-end.

During the initialization, the class constructor automatically creates the report file inside the specified folder tree, setting up its header and declaring the general options for the rmarkdown file. As soon as the analyst or the user proceeds with his analysis, the rmarkdown file is updated with a new CC each time the analysis software performs a new analysis step. When the analysis is complete, it is possible to compile the report using the compile() method, which produces the final report (in the user-defined format) and appends a final CC with the sessio$n$ Info to trace all the packages versions used for the analysis. Additionally, this process creates two others optional sections, one with the cited references when a bibliography file has been specified during the easyreporting instance creation, and another named Resources Availability with the resources specified with the addResource() method (see Section 3.9 in S1 File for more details).

\section{General exploitation}

The easyreporting class is equipped with several methods for rmarkdown CC construction (see Table 1 for the full list). Once an easyreporting instance is available, it is possible to organize the report by inserting up to six levels of titles by using the $m k d T i t l e$ method. It is also possible to add natural language comments with $m k d G e n e r a l M s g$. The latter feature is particularly relevant to make the analysis more understandable.

For the implementation of the CCs creation, we suggest two main approaches based on the methods available in the class (see the examples in Listings 2 and 3 shown in the Results Section): i) The first approach builds a CC as a typical step-by-step process. It consists of opening a CC ( $m k d C o d e C h u n k S t)$, adding variables assignments and/or function callings 

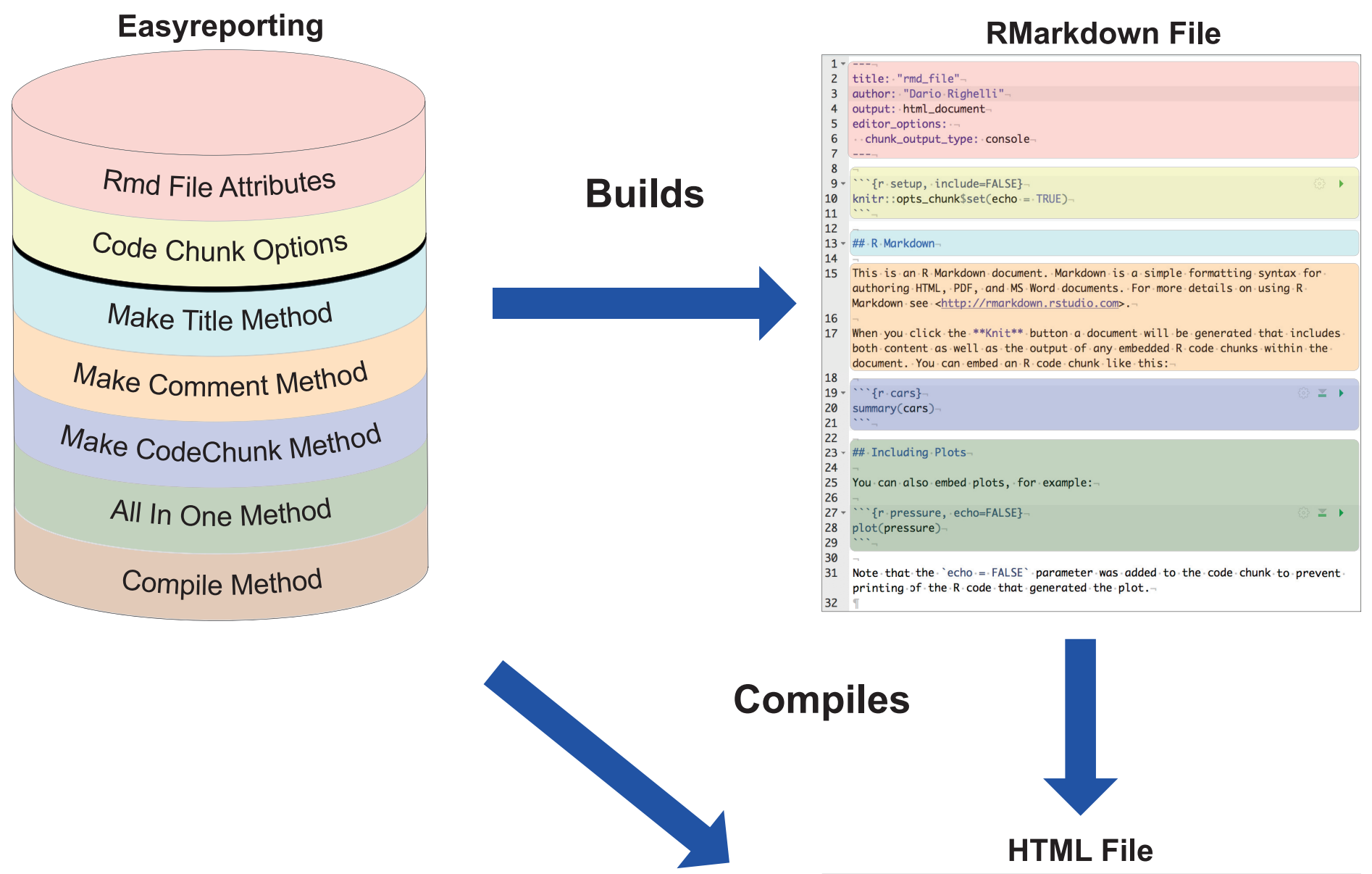

\section{Compiles}

Fig 1. The easyreporting class package is a representation of a rmarkdown file. The color codes indicate which attribute/method represents the same-color portion of the rmarkdown first, and the compiled final report then (in this example an HTML file).

https://doi.org/10.1371/journal.pone.0244122.g001 
Table 1. Attributes and methods of the EasyReporting class.

\begin{tabular}{|c|c|}
\hline Attributes & Description \\
\hline filenamePath & the report file name with the absolute path \\
\hline title & the title of the report \\
\hline author & the auhor \\
\hline documentType & actually this is set to HTML \\
\hline optionList & a list of R Markdown options \\
\hline bibfile & a latex bibliography filename \\
\hline resources & a data frame to store additional resources \\
\hline Methods & Description \\
\hline mkdTitle & Inserts an R Markdown title inside the report \\
\hline mkdGeneralMsg & appends a general message to the report \\
\hline mkdGeneralTitledMsg & appends a title and a general message to the report \\
\hline mkdVariableAssignment & includes a variable assignment into the report \\
\hline mkdCodeChunkSt & creates a CC start \\
\hline mkdCodeChunkEnd & creates a CC end \\
\hline mkdCodeChunkComplete & creates a complete CC \\
\hline mkdCodeChunkCommented & creates a complete CC with a previous comment \\
\hline mkdCodeChunkTitledCommented & creates a complete $\mathrm{CC}$ with a previous comment and a title \\
\hline mkdSourceFiles & includes a list of source files inside the CC \\
\hline compile & prints sessionInfo and compiles the R Markdown file \\
\hline setOptionsList & set an optionList to the class \\
\hline getOptionsList & returns the optionList from the class \\
\hline getReportFilename & returns the report filename with path \\
\hline getBibliography & returns the bibliography filename \\
\hline addResource & adds en entry into the references class data frame \\
\hline Exported Utility Functions & Description \\
\hline makeOptionsList & makes a list of rmarkdown options that can be passed to the class \\
\hline erGUIVolcano & executes a Shiny GUI to perform a volcano plot and trace its executed functions \\
\hline
\end{tabular}

https://doi.org/10.1371/journal.pone.0244122.t001

(mkdVariableAssignement), and finally closing the CC (mkdCodeChunkEnd). In this approach, it is possible to add comments with $m k d G e n e r a l M s g$ before closing the CC. ii) The second approach builds the CC in a single step by using the mkdCodeChunkComplete method. The method automatically embraces the tracking code into a new CC, while the user has to take care of the variable assignments and/or the function he/she wants to trace by passing it as an argument. In this approach, the user can also add personal comments passing them as an additional argument. Then, easyreporting automatically adds the comment before the new CC.

The first approach appears useful when one needs to carry out several $\mathrm{R}$ commands in a single CC. It is similar in the spirit to the functionalities offered by R-studio or other development environments, however since it is entirely command-line, it can be easily used on systems with limited development capabilities. By contrast, the second approach is more appropriate for tracing a single function call automatically. The next section will show how this second possibility can help wrap functions performing a specific step and trace their execution within GUIs or packages.

\section{Implementing automatical tracing functions and their usage within GUIs}

The previously described CCs creation approaches can be adapted to trace several steps of an analysis pipeline and end-up with a nicely formatted and detailed analysis report. However, 
they require the analysts to manually trace each step of the analysis (as he/she could also do with other available tools). Consequently, the above approaches are useful for generating analysis reports (that was the first aim of the easyreporting package). However, they are not suited for the automatic tracing of the steps of an analysis performed using packages function calling or point-and-click approaches through GUIs (the second aim of the easyreporting package).

In the last decade, GUIs are becoming very popular in bioinformatics because they simplify computational analysis allowing non-expert users to choose among several computational procedures, algorithms, and parameter settings, see for example [13, 14, 16-18]. In particular, the shiny (https://shiny.rstudio.com) libraries simplified the development of GUIs that incorporate the power of the statistical $\mathrm{R}$ language and the wide-amount of open-source packages available in repositories such as Bioconductor (https://www.bioconductor.org).

Nevertheless, computational studies obtained from GUIs might lack reproducibility since tracking all user choices is still challenging. To face this limit, the developers have to implement a RR layer when designing the GUI's back-end so that the final users can benefit from a better quality product. Moreover, the RR layer has to be transparent but understandable to not-expert users. Ideally speaking, at the end of the analysis, the user should have a humanreadable report analogous to the one obtained using command-line approaches.

Easyreporting methods can be easily adapted to support the automatic tracing of any given function by combining a rendering function that performs the required step with a wrapping function that traces its execution. The wrapper function (WF) needs an easyreporting instance, and the arguments of the function to be traced (TF). Then, the developer inserts the WF in the back-end of the interface (i.e., the server if the context of a GUI is implemented with the shiny library) in the TF place. The front-end of the interface (i.e., the UI with the shiny library) remains unchanged. When the user interacts with the interface to invoke the TF, the back-end will invoke the WF, which will call both the TF function of interest and trace its usage with all parameters. In brief, employing wrapper functions makes it possible to implement a reproducible research layer within the GUI without implementing all the tracing rmarkdown code. Listings 4-6 illustrate a specific case with a volcano plot, and Fig 2 schematically represents the entire workflow of information.

Note that WP functions can be useful for developers or advanced users also to generate novel R packages or simply novel R-functions that automatically trace their usage. In this way, at the price of an initial effort of writing wrapper functions, their usage will be automatically traced in any context.

\section{Results}

To better illustrate the capabilities of easyreporting (version 1.3.2 released with Bioconductor 3.13), we first show its usage for generating an analysis report in a case study concerning the analysis of RNA-seq data (see S1 File for details), then we illustrate how to implement a simple GUI that automatically traces the performed step with code and parameters choice to produce a report.

\section{Easyreporting for the creation of analysis report}

The RNA-seq data used in the example allows investigating the differences in CD8+ dendritic T-cells of the immune response of two different antibodies compared with control, see [19] for more details. We chose this illustrative example since it is well-known that the analysis of RNA-seq data can lack reproducibility [20]. The dataset contains the raw counts of 37991 genes and is composed of two replicates for each of the three conditions: DEC (fd-scaDEC-205 antibody samples); E2 (E2 antibody samples) and UNTR (control samples). For illustrative 


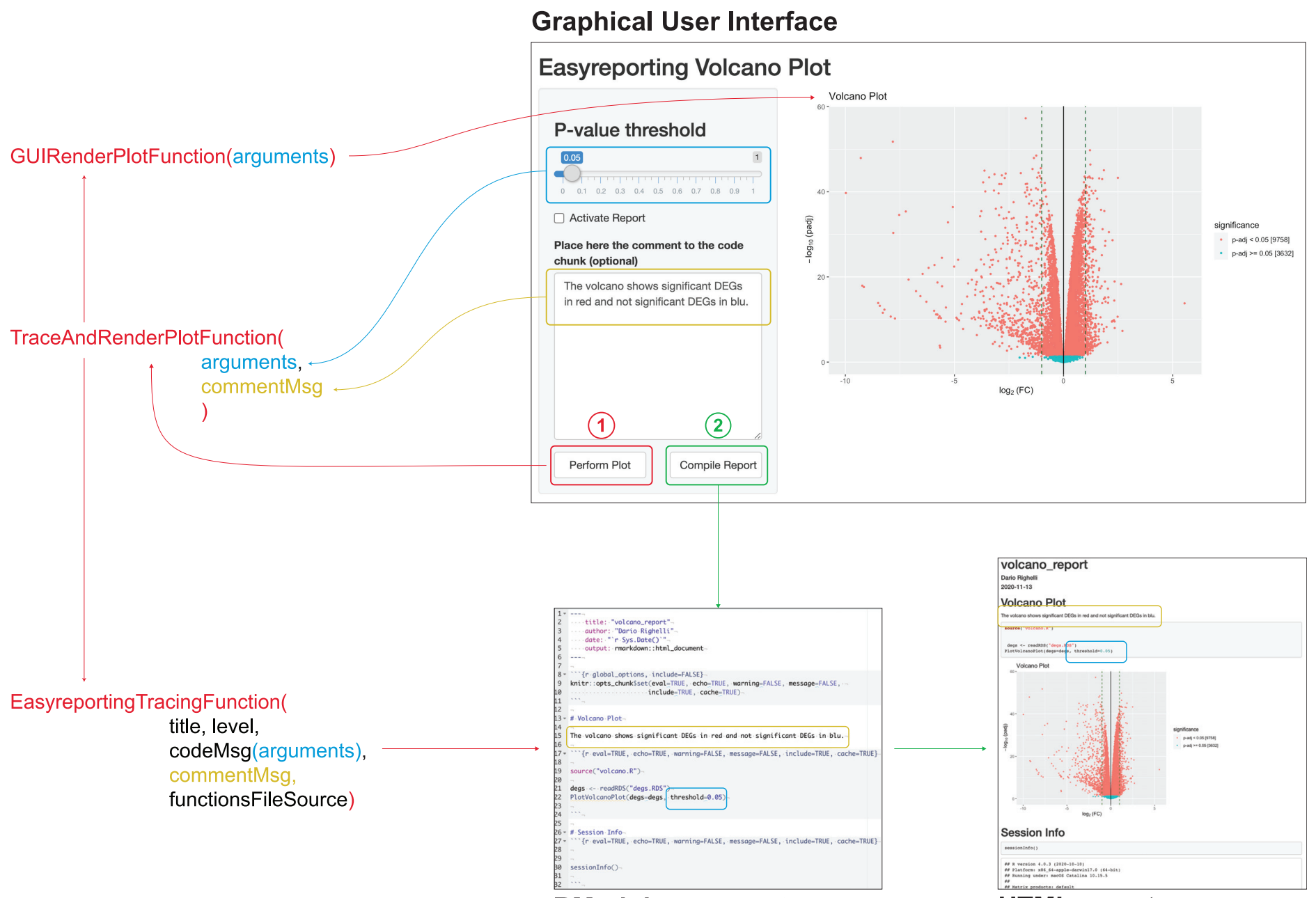

RMarkdown report

HTML report

Fig 2. Example of a graphical user interface working with easyreporting package.

https://doi.org/10.1371/journal.pone.0244122.g002

purposes, in our S1 File, we start the analysis from the raw count-matrix. Moreover, we released the raw counts as supplementary data with the easyreporting package, allowing the readers to reproduce our example. The naive pipeline will first load the data, perform some diagnostic plots, filter and normalize the raw counts, and visualize the principal component projection. It will then perform differential gene expression analysis and depict the results as a Venn diagram and MA-plots. A specific CC describes each phase.

In the following, we show the main fundamental steps that a user can adapt to any analysis, and we refer to S1 File for the detailed description of the remaining steps and S2 File for the complete report.

Report initialization. After loading the easyreporting package in the R environment, the analyst needs to initialize an analysis report by providing the file name (i.e., "rnaseq_report") and the title of the document (i.e., "RNA-seq Analysis Report"). It is also possible to specify one or more authors (i.e., "Dario Righelli") with additional associated information. For simplicity, we set-up a project directory path starting from the working directory for our report, but the user can choose other locations by setting the filenamepath parameter. The initialization is carried out by using the easyreporting() function. Note that the filenamepath and title 
are mandatory parameters, while the author(s) is optional. The following Listing 1 code illustrates the initialization of a report. We refer to S1 and S2 Files (chunk of code 1) for an example where multiple authors with affiliations and additional information are provided during the initialization.

Listing 1. Initialization chunk

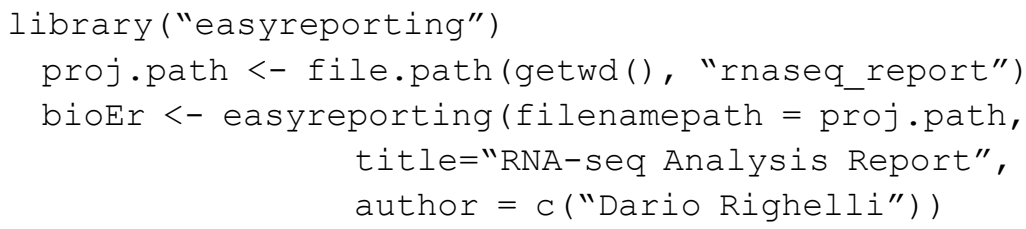

Creation of a chunk of code. Once the analyst has initialized the report, he/she can add a $\mathrm{CC}$ for each step of the analysis. As mentioned in the General Exploitation section, easyreporting provides two main approaches for adding CCs within a report: 1) building up the CC step by step (as shown in Listing 2) and 2) using several kinds of wrapper functions (as shown in Listing 3).

As mentioned above, in the first case, the analyst has to use the mkdCodeChunkSt to open a new CC. Then, he/she needs to add the code to markdown, by using the mkdVariableAssignment and/or the mkdGeneralMsg functions, for tracking variables and functions. Finally, the analyst has to close the CC using the mkdCodeChunkEnd function. The following Listing 2 code illustrates a step-by-step CC for loading the counts' matrix released with the package.

Listing 2. Step-by-step chunk construction

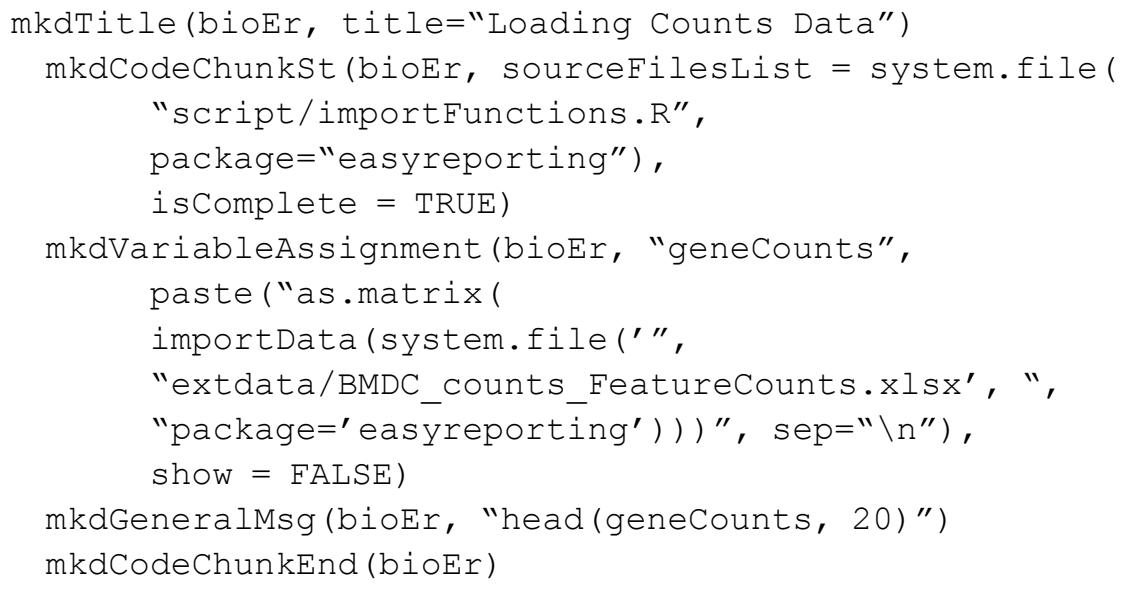

Although the first approach leaves complete freedom to the analyst, it can be tricky for small CCs. The second approach can be more straightforward for small CCs. To this purpose, the mkdCodeChunkComplete function allows tracing the steps through the message parameter. The following Listing 3 code illustrates an example of a single step CC. As for the above CC, we assume that the analyst wants to read the raw counts using a user-defined function, here named "importData.R", that we stored into the importFunctions.R file available in the package "script" folder. To simplify the code writing, we embraced the calls with the quote function, which allows passing raw code facilitating parenthesis highlighting and function recognition to the user (see Listing 3 for an example). Additionally, it is possible to pass multiple instruction as a list of quote with the $c()$ operator (see Section 3.3 in S1 File for an illustrative example).

Listing 3. One command chunk construction

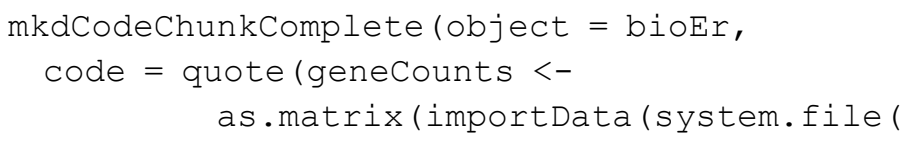




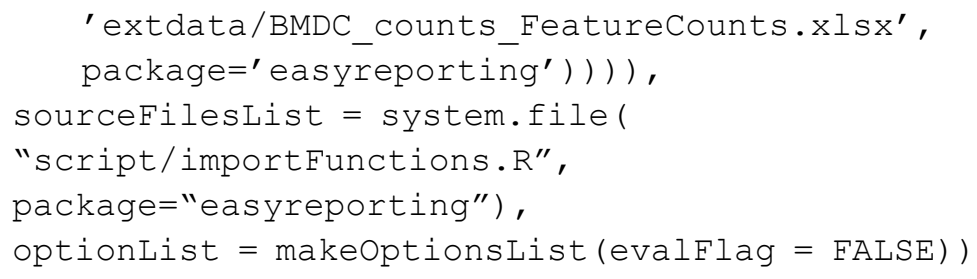

Note that the mkdCodeChunkComplete allows also to provide specific options for the CC that we are creating. In particular, in this case, turning the evalFlag to FALSE the code is not compiled during the final report construction.

It is possible to organize the report using the mkdTitle function. The user has to repeat this operation for each step of the analysis, as shown in S1 File. At the end of the process, it is possible to compile the easyreporting instance and obtain the analysis report as in S2 File in the user-defined format (default is HTML).

Implementing automatically tracing functions. This section shows a possible approach to encapsulate easyreporting methods into third-parties functions to trace the analysis step and execute the code automatically.

First of all, the developer has to write an $\mathrm{R}$ function that performs the analysis step of interest (such as the MAedgeRMAPlotEx function for rendering an MA-plot, in our example). Listings 4 shows a simple example of rendering function.

Listing 4. MA-plot rendering function

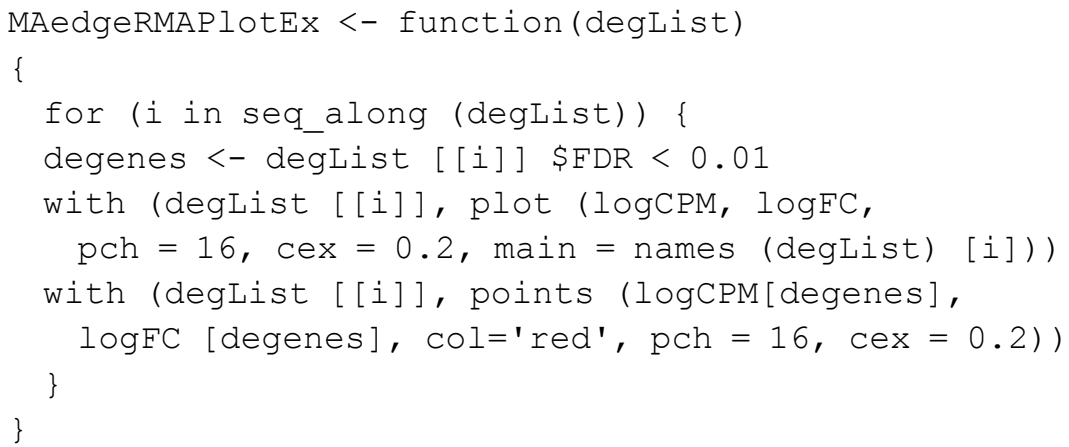

Note that the developer does not require any extra effort at this stage. Moreover, the rendering function could also be any function available from other packages.

Then, the developer needs also to write a wrapper function (here traceAndPlotMAPlot). The wrapper function should take as input the arguments of the rendering function (here MAedgeRMAPlotEx), and a generic easyreporting object (here er). Moreover, the wrapper function has to call the mkdCodeChunkTitledCommented function of easyreporting (where we insert the rendering function call to be traced (MAedgeRMAPlotEx) in the code argument) and the call to rendering function (MAedgeRMAPlotEx). Listings 5 shows the wrapper function of our example.

Listing 5. Tracing wrapper function chunk

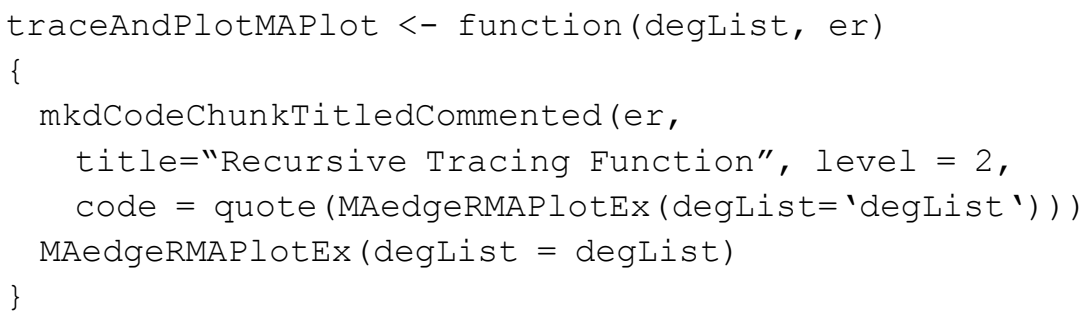

In this way, the wrapper function allows both to show the result and trace the function in the same step. It is easy to place the traceAndPlotMAPlot function-call wherever needed in the main code. 
Listing code 5 shows how to use the wrapper function. In particular, we pass as input i) the object required by the rendering function (an edgeR result class in this particular example), ii) an easyreporting class instance (here it is the bioEr instantiated in the example).

Listing 6. Tracing function call chunk

traceAndPlotMAPlot (degList = degList, er = bioEr)

Note that writing the wrapper function is the only extra effort required to achieve reproducibility. This approach can be used for writing user-friendly tools, novel R-packages or simply user-defined R-functions that automatically traces their usage.

\section{Easyreporting for GUI implementation}

To better illustrate how to incorporate a RR layer into a GUI, easyreporting contains a simple Shiny-App example for plotting a Volcano plot. The command erGUIVolcano() allows executing the app and opens the user interface. The user interface allows the user to choose a threshold for the P-value (i.e., the P-value threshold for detecting the significant genes in this example) and provide a text area for adding comments. In the interface, there are also two buttons (Perform Plot and Compile Report) for executing the plot and compiling the report, respectively. The ui function provides the code for the user interface. This code does not need to be modified to allow reproducibility. Instead, the back-end of the interface that executes the job has to incorporate a wrapper function (here traceAndPlotVolcano), and the call to the wrapper function, respectively. In this example, we also added the report's initialization. The server function provides the code for the back-end interface.

Fig 2 right side shows a schematic representation of the user interface as in the ui function. The left side illustrates the back-end as in the server function. By using the Perform Plot button (red box), the user activates the WF into the server-side, which in turn performs the plot and traces the executed function (the red cascade). In blue is highlighted the argument value and how it is traced through the function cascade.

Additionally, when the user adds its comments (yellow box) to the performed analysis step, the text is passed to the server.

Finally, the user can compile the report using the Compile Report button (green box). In this way, the server executes the compile() function and produces the HTML report that is automatically showed to the user.

This simple example can be generalized to complex interfaces to trace all user interactions.

\section{Conclusions}

Easyreporting can be used to support RR in different analysis contexts. However, it is particularly suited for analyzing omics data and developing software/GUIs, as we have shown in this work. Compared to other previously proposed solutions such as [21], that require not negligible commitments by the final user, potentially bringing him/her to renounce to include RR inside the scripts, the implementation of a RR layer with our approach is straightforward. Moreover, it leaves maximum freedom to the developer/analyzer for automatically creating and storing an rmarkdown document and providing methods for its compilation and adding comments in natural language. However, in omics data analysis, it is common to use mixed scenarios and combine different programming languages depending on the availability of specific methods and functionalities in the community or to integrate analysis with queries to online databases. Easyreporting can provide support also for these cases, at least up to a certain level. Using interface functions between $\mathrm{R}$ and other programming languages, we can execute in $\mathrm{R}$ a few steps of an analysis implemented in other languages. For example, thanks to the $\mathrm{R}$ package reticulate it is possible to run in R some Python functions. Similarly, using suitable 
API functions, we can easily interface $\mathrm{R}$ with on-line databases and execute the queries within the R environment. For example, using the R packages GEOquery or TCGAbiolinks, we can query and process data from the Gene Expression Omnibus (GEO) and the Genomic Data Commons (GDC) Data Portal, respectively. Nowadays, in the Bioconductor repository, many API packages allow interfacing the most common biological databases. By contrast, we should underline that if the analyses are not performed within the R environment, the user must manually document the external steps (for example, in the current release, using comments, adding.bib files, or linking to external resources as databases identifiers). Unfortunately, if the manual documentation is not detailed, the full reproducibility might be lost. Future releases of easyreporting will provide additional tools for handling the mixed case scenario.

Although several functionalities are already available, easyreporting can still benefit from some extra features such as methods for file editing, graphical representation of the analysis, and data caching. In particular, file editing can be useful for modifying specific CCs, and the graphical representation of the analysis can be useful to provide reports not only readable by third-party users but also graphically visualized as workflows. On the other hand, even though a dedicated data caching infrastructure can offer more manageability and share-ability of the data at the moment, it can be already performed in easyreporting by rmarkdown CCs option flag.

Finally, thanks to its versatility, easyreporting can be ideally included in any well-structured $\mathrm{R}$ project and the development of GUIs and packages, helping to fulfil most of the proposed rules in [2]. Moreover, if combined with virtualization solutions such as docker containers (i.e., docker4seq [22]) it helps to create fully reproducible projects. Easyreporting naturally complements docker containers in terms of reproducibility, allowing both the preserve code lines and user parameters and the computational environments and dependencies.

To conclude, our approach still requires the developer's effort to implement a RR layer into their software, which makes us imagine possible future works in this area where the code tracing is entirely left to the machine. The Java language provides a well-known example that uses the Aspect-Oriented Programming (AOP) paradigm for the software logging aspects. Unfortunately, this paradigm is still missing in the $\mathrm{R}$ language, but possible future approaches in the Reproducible Research area inside R could rely on implementing it, which, combined with rmarkdown or similar procedures, can be used to trace ad-hoc tagged functions and to log them into the report file. In such a way, reproducibility could be easier to implement and lesser subject to human errors.

\section{Supporting information}

S1 File. An illustrative example for the creation of an analysis report. (PDF)

S2 File. The report file obtained using the analysis steps described in S1 File. (PDF)

\section{Author Contributions}

Conceptualization: Dario Righelli, Claudia Angelini.

Data curation: Dario Righelli.

Formal analysis: Dario Righelli.

Funding acquisition: Claudia Angelini. 
Methodology: Dario Righelli, Claudia Angelini.

Software: Dario Righelli.

Supervision: Claudia Angelini.

Writing - original draft: Dario Righelli, Claudia Angelini.

Writing - review \& editing: Dario Righelli, Claudia Angelini.

\section{References}

1. Ioannidis JPA. Why most published research findings are false. In: Getting to Good: Research Integrity in the Biomedical Sciences; 2018.

2. Sandve GK, Nekrutenko A, Taylor J, Hovig E. Ten Simple Rules for Reproducible Computational Research; 2013.

3. Brito JJ, Li J, Moore JH, Greene CS, Nogoy NA, Garmire LX, et al. Recommendations to enhance rigor and reproducibility in biomedical research. GigaScience. 2020; 9(6):1-6. https://doi.org/10.1093/ gigascience/giaa056

4. Griffin PC, Khadake J, LeMay KS, Lewis SE, Orchard S, Pask A, et al. Best practice data life cycle approaches for the life sciences. F1000Research. 2018;. https://doi.org/10.12688/f1000research. 12344.2

5. Knuth DE. Literate Programming. The Computer Journal. 1984; 27(2):97-111. https://doi.org/10.1093/ comjnl/27.2.97

6. Russo F, Righelli D, Angelini C. Advantages and Limits in the Adoption of Reproducible Research and R-Tools for the Analysis of Omic Data. In: International Meeting on Computational Intelligence Methods for Bioinformatics and Biostatistics. Springer; 2015. p. 245-258.

7. Bailey DH, Borwein JM, Stodden V. Facilitating Reproducibility in Scientific Computing: Principles and Practice. In: Reproducibility: Principles, Problems, Practices, and Prospects; 2015.

8. Boettiger C. An introduction to Docker for reproducible research. In: Operating Systems Review (ACM); 2015.

9. Piccolo SR, Frampton MB. Tools and techniques for computational reproducibility; 2016.

10. Leisch F. Sweave: Dynamic Generation of Statistical Reports Using Literate Data Analysis. Compstat. 2002;.

11. Yihui Xie, J J Allaire GG. R Markdown: The Definitive Guide. Transforming Climate Finance and Green Investment with Blockchains. 2018; https://doi.org/10.1016/B978-0-12-814447-3.00041-0

12. Landau WM. The drake $R$ package: A pipeline toolkit for reproducibility and high-performance computing. Journal of Open Source Software. 2018; 3(21):550. https://doi.org/10.21105/joss.00550

13. Russo F, Angelini C. RNASeqGUI: A GUI for analysing RNA-Seq data. Bioinformatics. 2014;. https:// doi.org/10.1093/bioinformatics/btu308 PMID: 24812338

14. Russo F, Righelli D, Angelini C. Advancements in RNASeqGUI towards a Reproducible Analysis of RNA-Seq Experiments. BioMed Research International. 2016; 2016:11. https://doi.org/10.1155/2016/ 7972351 PMID: 26977414

15. Greenbaum D, Rozowsky J, Stodden V, Gerstein M. Structuring supplemental materials in support of reproducibility. Genome Biology. 2017; . https://doi.org/10.1186/s13059-017-1205-3 PMID: 28381262

16. Rue-Albrecht K, Marini F, Soneson C, Lun AT. iSEE: interactive summarizedexperiment explorer. F1000Research. 2018; 7. https://doi.org/10.12688/f1000research.14966.1 PMID: 30002819

17. Criscuolo NG, Angelini C. StructuRly: A novel shiny app to produce comprehensive, detailed and interactive plots for population genetic analysis. Plos one. 2020; 15(2):e0229330. https://doi.org/10.1371/ journal.pone.0229330 PMID: 32074134

18. Di Filippo L, Righelli D, Gagliardi M, Matarazzo MR, Angelini C. HiCeekR: a novel Shiny app for Hi-C data analysis. Frontiers in genetics. 2019; 10:1079. https://doi.org/10.3389/fgene.2019.01079 PMID: 31749839

19. Costa V, Righelli D, Russo F, De Berardinis P, Angelini C, D'Apice L. Distinct antigen delivery systems induce dendritic cells' divergent transcriptional response: New insights from a comparative and reproducible computational analysis. International Journal of Molecular Sciences. 2017; 18(3):494. https:// doi.org/10.3390/ijms18030494 PMID: 28245601

20. Simoneau J, Dumontier S, Gosselin R, Scott MS. Current RNA-seq methodology reporting limits reproducibility. Briefings in Bioinformatics. 2019; https://doi.org/10.1093/bib/bbz124 
21. Napolitano F. repo: An R package for data-centered management of bioinformatic pipelines. BMC Bioinformatics. 2017; 18(1):112. https://doi.org/10.1186/s12859-017-1510-6 PMID: 28209127

22. Kulkarni N, Alessandrì L, Panero R, Arigoni M, Olivero M, Ferrero G, et al. Reproducible bioinformatics project: A community for reproducible bioinformatics analysis pipelines. BMC Bioinformatics. 2018; 19 (10):211. https://doi.org/10.1186/s12859-018-2296-x PMID: 30367595 